\title{
ON GEOMETRY OF FRONTS IN WAVE PROPAGATIONS
}

\author{
SUSUMU TANABÉ \\ Max Planck Institut für Mathematik \\ Vivatsgasse 7, D-53111 Bonn, Germany \\ Independent University of Moscow \\ Bol'shoŭ Vlas'evskiน Pereulok 11, 121002 Moscow, Russia \\ E-mail:tanabe@mpim-bonn.mpg.de,tanabe@mccme.ru
}

\begin{abstract}
We give a geometric descriptions of (wave) fronts in wave propagation processes. Concrete form of defining function of wave front issued from initial algebraic variety is obtained by the aid of Gauss-Manin systems associated with certain complete intersection singularities. In the case of propagations on the plane, we get restrictions on types of possible cusps that can appear on the wave front.
\end{abstract}

0. Introduction. In this note, we intend to develop a preparatory theory toward asymptotic analysis around singular loci that appear in wave propagation process with initial data whose $C^{\infty}$ singularities are located on certain smooth algebraic variety. For this purpose, we study the geometry of the (wave) front, i.e. the singular support S.S. of the solutions to the Cauchy problem where it loses $C^{\infty}$ smoothness. We give a concrete expression of defining function of the wave front in terms of the initial data (Theorem 10). We impose conditions on the initial data so that they are defined by a quasihomogeneous polynomial with isolated singularities. The key trick is to understand the (wave) front as a discriminantal set for a deformation of complete intersection singularity. This point of view has been exposed in [20].

As is well known, the fundamental solutions of hyperbolic operators are expressed by means of certain kinds of Gel'fand-Leray integrals. Thus the geometry of singular support (i.e. front) of the solutions is reduced to the study of singular support of these types of integrals. Homological and cohomological approach to the analysis of sharp-diffuse type of these integrals has been initiated by [3]. V. A. Vassiliev [21] uses F. Pham's approach [15] analyzing homology classes to describe the ramification character of certain kinds of Gel'fand-Leray integrals, consequently to clarify the sharp-diffuse type (see Section 4 for

1991 Mathematics Subject Classification: Primary 35L25, 58G17, 33C75; Secondary 32S40, 78A05, 33C20.

The paper is in final form and no version of it will be published elsewhere. 
the definition) of the solutions to hyperbolic operators in the neighbourhood of singular loci of the wave front, under the condition that one knows the type of singularities of the singular loci in question. Our method furnishes such existing results with a supplementary tool while we derive informations, global in space and time, uniquely from the initial data. In the sense that global behaviour of solutions to Cauchy problem is investigated, this paper is a continuation of [16], in which a similar setting of question has been studied by means of analysis of lagrangian variety.

To pursue further this study, we propose to make use of the Gauss-Manin system associated with fibre bundle structure that naturally arises in integration. Our main tool is concrete expressions of the overdetermined differential systems obtained from nontrivial relations between base elements of a Brieskorn lattice for complete intersection singularities (Proposition 5, Theorem 7).

Theorem 11 and the illustration given in Section 4 serve as a motivation why we propose to carry out asymptotic analysis by means of the Gauss-Manin systems. They justify our proposition in the sense that they demonstrate the possibility to interpret solutions to the Gauss-Manin systems as generalized hypergeometric functions. This procedure is supported by the general theory of the Gauss-Manin systems for isolated complete intersection singularities [6]. More systematic explanation of this situation from singularity theoretical point of view is given in [1] and [19].

Section 3 illustrates how one can apply the theory developed in Section 2 to situations on the plane. Our Theorem 12 says that cusps that can appear on wave fronts issued from an initial front defined by a polynomial of degree $k$ are at worst $(2 k-1,8 k-2)$. We do not come into a detailed study of all possible cusps in terms of coefficients $\left(g_{1}, \ldots, g_{k-1}\right)$ of the defining polynomial. After the establishment of an algorithm, such kind of detailed study can be achieved by anybody who tries to write down conditions like that in Remark 1.

1. Preliminaries on the Cauchy problem. In this section we prepare fundamental notation and lemmata to develop our studies in further sections. First, let us regard the following Cauchy problem:

$$
\left\{\begin{aligned}
{\left[D_{t}^{2}-\Delta_{x}\right] u(t, x) } & =0 \\
D_{t} u(0, x) & =v(x) \\
u(0, x) & =0
\end{aligned}\right.
$$

where $t \in \mathbf{R}, x \in \mathbf{R}^{n}, D_{t}=\frac{\partial}{i \partial t}, \Delta_{x}=\sum_{j=1}^{n}\left(\frac{\partial}{i \partial x_{j}}\right)^{2}, i=\sqrt{-1}$. We will study the Cauchy problem (C.P.) under the conditions (C.1), (C.2), (C.3) formulated below, imposed on the initial data.

In order to describe these conditions, we use the notation $\chi_{q}^{\epsilon}(z)$ (where $\epsilon= \pm 1$ ) which stands for the following distributions:

$$
\chi_{q}^{\epsilon}(z)=\chi_{q}(z+i 0)+\epsilon \chi_{q}(z-i 0),
$$

where

$$
\begin{array}{ll}
\chi_{q}(z)=z^{q} / \Gamma(q), & q \notin \mathbf{Z} \text { or } q \text { negative integer }, \\
\chi_{q}(z)=\frac{z^{q}}{q}\left(-\log z+C_{q}\right), & q \text { positive integer }
\end{array}
$$


and $C_{0}=0, C_{q}=C_{q-1}+1 / q$. Remark that

$$
\frac{d}{d z} \chi_{q}(z)=\chi_{q-1}(z)
$$

(C.1) The initial data are given by a distribution of finite order with singular support (see Definition 2.2.3 of [10]) located on cotangent bundle of a smooth algebraic surface $S:=\left\{x \in \mathbf{R}^{n}: F(x)-s=0\right\}$ defined by a real polynomial $F(x)$,

$$
v(x)=g(x) \chi_{q}^{\epsilon}(F(x)-s)
$$

with a smooth function $g(x)$.

Further we shall denote the singular support of a distribution $v(x)$ by S.S.v $(x)$.

We impose several technical conditions also. These conditions will be used so that the reasoning on the isolated complete intersection singularities can be applied to our (C.P.)

(C.2) (Quasihomogeneity) There exists a set of integers $\left(w_{1}, \ldots, w_{n}\right)$ which satisfies: 1) $w_{i} \neq w_{j}$ for a certain pair $\left.1 \leq i \neq j \leq n, 2\right)$ G.C.D. $\left(w_{1}, \ldots, w_{n}\right)=1$ and 3 ) for $a$ positive integer $w(F)$,

$$
\left(\sum_{1 \leq j \leq n} w_{j} x_{j} \frac{\partial}{\partial x_{j}}\right) F(x)=w(F) F(x) .
$$

(C.3) The following $\mathbf{R}[x]$-module is of finite dimension:

$$
\mathbf{R}[x] / \mathcal{I}
$$

where $\mathcal{I}=\left\langle F(x), \frac{\partial F}{\partial x_{1}}(x), \ldots, \frac{\partial F}{\partial x_{n}}(x)\right\rangle$ (ideal generated by the entries).

Let us introduce the following notation.

a) The phase function $\psi(x, t, z)$ is defined as follows:

$$
\psi(x, t, z)=\left\langle x-z, \operatorname{grad}_{z} F(z)\right\rangle^{2}-t^{2}\left|\operatorname{grad}_{z} F(z)\right|^{2} .
$$

b) The paired oscillatory integrals introduced in [5] which are defined for the phase function $\psi(x, t, z)$ of $\mathbf{a})$ :

$$
I_{p}^{\epsilon}(x, t, s)=\int_{\{F(z)=s\}} H_{p}(z) \chi_{p}^{\epsilon}(\psi(x, t, z)) \frac{d z}{d F},
$$

with regular amplitude functions of the pseudo-differential operator

$$
H_{p}(z) \sim \sum_{r=p-2}^{-\infty} h_{p, r}(z) \in S^{p-2}\left(\mathbf{R}^{n}\right),
$$

in which $h_{p, r}(z)$ is homogeneous of order $r$ for large values of $z$. One will understand $I_{p}^{\epsilon}(x, t, s)$ as the Gel'fand-Leray integral (see $[4,1.5]$ ) defined on the real algebraic set $S=\left\{z \in \mathbf{R}^{n}: F(z)=s\right\}$.

c) The function $\phi(x, t, s)$ is the defining function of the (wave) front $\Sigma$ issued from $S$ determined by (C.P.). 
Proposition 1. We use the notation introduced in $\mathbf{a}), \mathbf{b})$ and $\mathbf{c})$ above.

d) The solution to the Cauchy problem (C.P.) $u(x, t)$ admits an asymptotic expansion

$$
u(x, t) \sim \sum_{j=0}^{\infty} I_{-n / 2+q+j}^{\epsilon}(x, t, s) .
$$

That is to say, for every $N \gg 0$ there exists $C_{N}>0$ such that

$$
\left|u(x, t)-\sum_{j=0}^{N} I_{-n / 2+q+j}^{\epsilon}(x, t, s)\right| \leq C_{N}|\phi(x, t, s)|^{q+N+1}
$$

in the neighbourhood of S.S.u $(x, t)$.

P r o of. We give only a sketch of proof while a detailed one will appear in [19]. First of all we show that the phase function of the integrals $I_{p}^{\epsilon}(x, t, s)$ coming into a) is given by $\mathbf{c})$.

Solving the Hamilton-Jacobi equation associated with the Hamiltonian $\tau \pm|\xi|$ (in a symplectic coordinate with canonical symplectic form $d t \wedge d \tau+\sum_{j=1}^{n} d x_{j} \wedge d \xi_{j}$; for the symplectic geometry see [10, Chapter XXI]),

$$
\left\{\begin{aligned}
\dot{t} & =1 \\
\dot{\tau} & =0 \\
\dot{x}_{j} & = \pm \frac{\partial|\xi|}{\partial \xi_{j}} \\
\dot{\xi}_{j} & =0 \\
x_{j}(0) & =z_{j},\left\{z \in \mathbf{R}^{n}: F(z)=s\right\}, 1 \leq j \leq n
\end{aligned}\right.
$$

we get

$$
x_{j}= \pm t \frac{\partial|\xi|}{\partial \xi_{j}}+z_{j}= \pm t \frac{\xi_{j}}{|\xi|}+z_{j} \quad \text { with } \quad z \in S=\left\{z \in \mathbf{R}^{n}: F(z)=s\right\} .
$$

This means that the singularities of the solutions to (C.P.) lie on the rays (1.3). These lines are interpreted as rays issued from the initial front $S=\left\{z \in \mathbf{R}^{n}: F(z)=s\right\}$ in the direction determined by the Hamiltonians $\tau^{2}-|\xi|^{2}$. Consequently they are expressed by integrals with phase

$$
\psi(x, t, z)=\left\langle x-z, \operatorname{grad}_{z} F(z)\right\rangle^{2}-t^{2}\left|\operatorname{grad}_{z} F(z)\right|^{2} .
$$

Here we remark that for every $p \in \mathbf{Q}$ and $H(z) \in \mathcal{D}^{\prime}\left(\mathbf{R}_{z}^{n}\right)$, we have

$$
\left[D_{t}^{2}-\Delta_{x}\right] \int_{S} H(z)(\psi(x, t, z))^{p} \frac{d z}{d F}=0 .
$$

One can prove this equality with the aid of Gauss-Ostrogradskii-Stokes' theorem. Thus the question is how to find a series of integrals

$$
I_{p}^{\epsilon}(x, t, s)=\int_{S} H_{p}(z) \chi_{p}^{\epsilon}(\psi(x, t, z)) \frac{d z}{d F}, \quad p \in \mathbf{Q},
$$

whose suitably converging sum produces a distribution $u(x, t)$ satisfying (C.1). The possibility of an asymptotic expansion (1.2) consisting of terms like $\mathbf{b}$ ) can be proven by well known estimates on the stationary phase ([10, Theorem 7.7.12]). More precisely, let us remind the following lemma. 
LEMMA 2. Let $(G)^{0}(x, t, s)$ be a residue of a smooth function $G(x, t, z)$ after division by an Jacobi ideal generated by $\frac{\partial \psi(x, t, z)}{\partial z_{j}}, \frac{\partial F(z)}{\partial z_{j}}, 1 \leq j \leq n$, and $F(z)-s$, i.e.

$$
\begin{aligned}
G(x, t, z)=G^{0}(x, t, s)+\sum_{j=1}^{n} & f_{j}(x, t, s, z) \frac{\partial \psi(x, t, z)}{\partial z_{j}} \\
& +\sum_{j=1}^{n} g_{j}(x, t, s, z) \frac{\partial F(z)}{\partial z_{j}}+h(x, t, s, z)(F(z)-s),
\end{aligned}
$$

with some smooth functions $h(x, t, s, z), f_{j}(x, t, s, z), g_{j}(x, t, s, z), 1 \leq j \leq n$.

Then for every smooth function $a(z)$ the following asymptotic estimate with some $C_{N}>0$ holds in the neighbourhood of the wave front $\Sigma=\left\{(x, t) \in \mathbf{R}^{n+1}:(\psi)^{0}(x, t, s)=0\right\}$ :

$$
\begin{array}{r}
\left|\int_{S} a(z) \chi_{q}^{\epsilon}(\psi(x, t, z)) d z-\sum_{j=0}^{N}\left(L_{\psi, j} a\right)^{0}(x, t, s) \chi_{q+n / 2+j}^{\epsilon}\left((\psi)^{0}(x, t, s)\right)\right| \\
<C_{N}\left|(\psi)^{0}(x, t, s)\right|^{n / 2+N+1+q}
\end{array}
$$

with differential operators $L_{\psi, j}$ of degree $2 j$. Furthermore we have

$$
\left(L_{\psi, 0} a\right)^{0}(x, t, s)=i^{n / 2}(2 \pi)^{(n-1) / 2}(a)^{0}(x, t, s)\left|\operatorname{det}\left(\frac{\psi_{z z}}{2 \pi i}\right)^{0}(x, t, s)\right|^{-1 / 2} .
$$

Let us briefly sketch the proof of the lemma. Malgrange's division theorem yields the decomposition (1.6) in connexion with the fact that the following $\mathcal{O}_{\mathbf{C}^{n} \text {-module is a }}$ finite-dimensional vector space under assumption (C.3):

$$
\frac{\Omega_{\mathbf{C}^{n}}^{n}}{d F(z) \wedge \Omega_{\mathbf{C}^{n}}^{n-1}+d \psi(0,0, z) \wedge \Omega_{\mathbf{C}^{n}}^{n-1}+d_{z} \Omega_{\mathbf{C}^{n}}^{n-1}+F(z) \wedge \Omega_{\mathbf{C}^{n}}^{n}} .
$$

Further it suffices to apply the stationary phase method mentioned above.

Following Lemma 2 the function $(\psi)^{0}(x, t, s)$ given by $(1.5)$ for $G(x, t, z)=\psi(x, t, z)$ in (1.4) can be considered as the defining function $\phi(x, t, s)$ of the wave front issued from $S$ determined by the Hamiltonian flow (1.3). The initial condition means $S=\{x: \phi(x, 0, s)=0\}$ which in turn entails $\mathbf{c})$.

It remains to justify asymptotic estimates in $\mathbf{b}$ ) and $\mathbf{d}$ ). This can be achieved in view of (1.6) and well known construction of an elementary solution to strictly hyperbolic Cauchy problem (see for example [8], [9]). Hence the assertion follows.

We formulate a simple lemma before introducing necessary notation.

Lemma 3. Under the assumptions (C.2), (C.3) imposed on $F(x)$ there exists a collection of at most quadratic polynomials $W_{1}(x, t), \ldots, W_{m}(x, t)$, with $m$ integer smaller than $2^{n} \prod_{i=1}^{n}\left(\frac{1}{w_{i}}\right)$, satisfying

$$
\psi(x, t, z)=\left\langle z, \operatorname{grad}_{z} F(z)\right\rangle^{2}+\sum_{i=1}^{m} W_{i}(x, t) z^{\alpha^{(i)}}
$$

for $\psi(x, t, z)$ of (1.4). Here $\alpha^{(i)}=\left(\alpha_{1}^{(i)}, \ldots, \alpha_{n}^{(i)}\right) \in\left(\mathbf{Z}_{\geq 0}\right)^{n}$ stands for multi-index under the restriction $\sum_{j=1}^{n} w_{j} \alpha_{j}^{(i)}<2$. 
The proof is a direct consequence of (1.4). The quasihomogeneous type of $F(z)$ yields the estimate on term number $m$.

Let us denote by

$$
w\left(z^{\alpha^{(i)}}\right)=\sum_{j=1}^{n} w_{j} \alpha_{j}^{(i)}
$$

the quasihomogeneous weight of the monomial $z^{\alpha^{(i)}}$ for $\alpha^{(i)} \in \mathbf{N}^{n}$. In terms of the quasihomogeneous weight we distinguish two cases.

Case 1. If there is a term with $w\left(z^{\alpha^{(i)}}\right)=0$, let us mark it as $\alpha^{(1)}$ and define the polynomial

$$
f_{1}(y(x, t), z)=\left\langle z, \operatorname{grad}_{z} F(z)\right\rangle^{2}+\sum_{i=1}^{m} y_{i}(x, t) z^{\alpha^{(i)}} .
$$

Here $y_{i}(x, t)=W_{i}(x, t), 1 \leq i \leq m$, for polynomials introduced in Lemma $3,(1.7)$.

Case 2. If all terms of (1.7) have positive weight, we define

$$
f_{1}(y(x, t), z)=\left\langle z, \operatorname{grad}_{z} F(z)\right\rangle^{2}+\sum_{i=2}^{m+1} y_{i}(x, t) z^{\alpha^{(i-1)}}+y_{1}
$$

with $y_{i+1}(x, t)=W_{i}(x, t), 1 \leq i \leq m$.

For the sake of simplicity we adopt the notation $\mu=m$ for Case 1 and $\mu=m+1$ for Case 2.

Further we define the integrals

$$
I_{p}(y(x, t), s)=\int_{S} H_{p}(z) \chi_{p}\left(f_{1}(y(x, t), z)\right) \frac{d z}{d F} .
$$

Hence if one denotes $y^{\prime}=\left(y_{2}(x, t), \ldots, y_{\mu}(x, t)\right)$, then

$$
I_{p}^{\epsilon}(x, t, s)=I_{p}\left(y_{1}+i 0, y^{\prime}(x, t), s\right)+\epsilon I_{p}\left(y_{1}-i 0, y^{\prime}(x, t), s\right)
$$

on understanding that the boundary value is taken at $y_{1}=0$ in Case 2 mentioned above. Thus it is essential to study $I_{p}(y(x, t), s)$ of (1.8) to estimate asymptotic behaviour of $I_{p}^{\epsilon}(x, t, s)$. From now on we shall regard the integral (1.8) as a function in variables $y(x, t)=\left(y_{1}, y_{2}(x, t), \ldots, y_{\mu}(x, t)\right)$. Therefore our main concern will be to investigate the differential equations that satisfy $I_{p}(y, s)$ corresponding to various amplitudes $H_{p}(z)$ with the aid of Gauss-Manin connexions associated to complete intersection singularities.

2. Gauss-Manin connexions for quasihomogeneous complete intersections. Here we propose to study the integrals $I_{p}(y, s)$ defined in $(1.8)$ by means of the GaussManin system associated with complete intersection singularities. In effect, it is well known that the Gauss-Manin connexion can be defined on the relative de Rham cohomology groups. Instead of that here we propose to calculate it on spaces of so called Brieskorn lattices (see [4], [6]).

The formulation of this section is a modification of $[17, \S 1]$ adapted to our situation. Let us observe a mapping between complex manifolds $X=\left(\mathbf{C}^{N+K}, 0\right), Y=\left(\mathbf{C}^{K}, 0\right)$,

$$
f: X \rightarrow Y
$$


that defines an isolated quasihomogeneous complete intersection singularity at the origin. That is to say, if we define

$$
X_{y}:=\left\{u \in X: f_{0}(u)=y_{0}, \ldots, f_{K-1}(u)=y_{K-1}\right\},
$$

then $\operatorname{dim} X_{y}=N \geq 0$ and the critical set of mapping $f: X_{0} \rightarrow Y$ is isolated in $X_{0}$. Further we assume that the polynomials $f_{0}(u), \ldots, f_{K-1}(u)$ are quasihomogeneous, i.e. there exists a collection of integers $v_{1}, \ldots, v_{N+K}$ whose greatest common divisor equals 1 and

$$
\left(v_{1} u_{1} \frac{\partial}{\partial u_{1}}+\ldots+v_{N+K} u_{N+K} \frac{\partial}{\partial u_{N+K}}\right) f_{\ell}(u)=p_{\ell} f_{\ell}(u), \quad \ell=0,1, \ldots, K-1,
$$

for certain integers $p_{0}, \ldots, p_{K-1}$. We shall call the vector field

$$
E=\sum_{i=1}^{N+K} v_{i} u_{i} \frac{\partial}{\partial u_{i}}
$$

Euler vector field, and $v_{1}, \ldots, v_{N+K}$ (resp. $p_{0}, \ldots, p_{K-1}$ ) weights of variables $u_{1}, \ldots$, $\ldots, u_{N+K}$ (resp. polynomials $f_{0}, \ldots, f_{K-1}$ ), i.e. $v_{1}=w\left(u_{1}\right), p_{0}=w\left(f_{0}\right)$ etc.

In order to calculate the Gauss-Manin connexion for isolated complete intersection singularity $X_{0}$, we introduce two vector spaces $V$ and $F$. After Greuel-Hamm [7], we look at a space whose dimension as a vector space over $\mathbf{C}$ is known to be the Minor number $\mu\left(X_{0}\right)$ of singularity $X_{0}$,

$$
V:=\frac{\Omega_{X}^{N}}{d f_{0} \wedge \Omega_{X}^{N-1}+\ldots+d f_{K-1} \wedge \Omega_{X}^{N-1}+d \Omega_{X}^{N-1}+f_{0} \Omega_{X}^{N}+\ldots+f_{K-1} \Omega_{X}^{N}} .
$$

The second one will later turn out to be isomorphic to $V$ (see Proposition 6 ),

$$
F:=\frac{\Omega_{X}^{N+1}}{d f_{0} \wedge \Omega_{X}^{N}+\ldots+d f_{K-1} \wedge \Omega_{X}^{N}+i_{E}\left(\Omega_{X}^{N+2}\right)} .
$$

Here $i_{E}$ means the inner contraction with Euler field $E$ defined by (2.2). The third vector space associated with the singularity $X_{0}$ is defined as follows

$$
\Phi:=\frac{\Omega_{X}^{N+K}}{d f_{0} \wedge \ldots \wedge d f_{K-1} \wedge \Omega_{X}^{N}+f_{0} \Omega_{X}^{N+K}+\ldots+f_{K-1} \Omega_{X}^{N+K}} .
$$

Later we define period integrals as coupling of forms of $V$ or of $\Phi$ with base element of homology groups $H_{N}\left(X_{y}\right)$. We remember also the definition of the Brieskorn lattice " $H$ from $[6]$,

$$
{ }^{\prime \prime} H=\frac{\Omega_{X}^{N+K}}{d f_{0} \wedge \ldots \wedge d f_{K-1} \wedge d \Omega_{X}^{N-1}},
$$

whose rank as $\mathbf{O}_{S}$-module equals the Minor number $\mu\left(X_{0}\right)$ of the singularity $X_{0}$.

It is easy to show

Lemma 4. For quasihomogeneous polynomials $f_{0}, \ldots, f_{K-1}$ defining an isolated complete intersection singularity,

$$
\Phi \cong{ }^{\prime \prime} H /\left(f_{0}, \ldots, f_{K-1}\right)
$$

Thus $\operatorname{dim}_{\mathbf{C}} \Phi=\mu\left(X_{0}\right)$. 
From definitions (2.4) and (2.5) we easily deduce

Proposition 5. For every form $\tilde{\omega}_{i} \in F$, one has the decomposition

$$
\tilde{\omega}_{i} \wedge d f_{0} \wedge \ldots{ }^{\vee} \wedge d f_{K-1}=\sum_{j=1}^{\mu\left(X_{0}\right)} P_{i j}^{(\ell)} \phi_{j}(u) d u \bmod \left(d f_{0} \wedge \ldots \wedge d f_{K-1} \wedge d \Omega_{X}^{N-1}\right)
$$

with $P_{i j}^{(\ell)} \in \mathbf{C}\left[f_{0}, \ldots, f_{K-1}\right]$ and $\phi_{j}(u) d u \in \Phi$, for $1 \leq i, j \leq \mu\left(X_{0}\right), 0 \leq \ell \leq K-1$ and $d f_{1} \wedge \ldots \vee \wedge f_{K-1}=\bigwedge_{i \neq \ell} d f_{i}$.

From [17] we remember

Proposition 6. Under the situation and definitions as above, the mapping

$$
i_{E}: F \rightarrow V
$$

induces an isomorphism. Consequently $\operatorname{dim}_{\mathbf{C}} F=\operatorname{dim}_{\mathbf{C}} V=\mu\left(X_{0}\right)$.

In view of Proposition 6 , let us denote the base of $V$ by $\left\{\omega_{i}\right\}$ where $\omega_{i}=i_{E}\left(\tilde{\omega}_{i}\right)$, $\tilde{\omega}_{i} \in F, 1 \leq i \leq \mu\left(X_{0}\right)$.

To make a transition from $(N+K)$-forms to period integrals, we introduce meromorphic $N$-forms $\psi_{i}$ satisfying

$$
d f_{0} \wedge \ldots \wedge d f_{K-1} \wedge \psi_{i}=\phi_{i}(u) d u, \quad 1 \leq i \leq \mu\left(X_{0}\right) .
$$

Then we derive the following relation from Proposition 6 :

$$
\begin{aligned}
& d \omega_{j}=\left(d i_{E}+i_{E} d\right)\left(\tilde{\omega}_{j}\right) \equiv \ell_{j} \tilde{\omega}_{j} \equiv \ell_{j}\left(\sum_{q=1}^{\mu\left(X_{0}\right)} P_{j q}^{(0)} d f_{0} \wedge \psi_{q}+\ldots\right. \\
& \left.+(-1)^{K-1} \sum_{q=1}^{\mu\left(X_{0}\right)} P_{j q}^{(K-1)} d f_{K-1} \wedge \psi_{q}\right) \bmod \left(\left(d f_{0}, \ldots, d f_{K-1}\right) d \Omega_{X}^{N-1}\right),
\end{aligned}
$$

where $\ell_{j}$ denotes the weight of the form $\omega_{j}$. Hence,

$$
\begin{aligned}
\omega_{j}=i_{E}\left(\tilde{\omega}_{j}\right) \equiv \sum_{i=0}^{K-1}(-1)^{i}\left[\sum_{q=1}^{\mu\left(X_{0}\right)} P_{j q}^{(i)} p_{i} f_{i} \psi_{q}-\sum_{q=1}^{\mu\left(X_{0}\right)} P_{j q}^{(i)} d f_{i} \wedge i_{E}\left(\psi_{q}\right)\right] \\
\bmod \left(\left(d f_{0}, \ldots, d f_{K-1}\right) i_{E} d \Omega_{X}^{N-1},\left(f_{0}, \cdots, f_{K-1}\right) d \Omega_{X}^{N-1}\right) .
\end{aligned}
$$

As a consequence

$$
\begin{aligned}
d \omega_{j}=\sum_{q=1}^{\mu\left(X_{0}\right)}[ & \left.\sum_{i=0}^{K-1}(-1)^{i}\left(d\left(p_{i} P_{j q}^{(i)} f_{i}\right)-w\left(\psi_{q}\right) P_{j q}^{(i)} d f_{i}\right)\right] \wedge \psi_{q} \\
& +\sum_{q=1}^{\mu\left(X_{0}\right)}\left[\sum_{i=0}^{K-1}(-1)^{i} p_{i} P_{j q}^{(i)} f_{i}\right] \wedge d \psi_{q} \bmod \left(\left(d f_{0}, \ldots, d f_{K-1}\right) d \Omega_{X}^{N-1}\right),
\end{aligned}
$$

where $w\left(\psi_{q}\right)$ is the quasihomogeneous weight of the form $\psi_{q}$. The expression (2.9) can be simplified if one lets them couple with a vanishing $N$-cycle, say $\gamma(y)$, and attains nontrivial relations between integrals $\int_{\gamma(y)} \psi_{q}$, instead of those between forms. One defines 
so called period integral $I_{\phi_{q}, \gamma(y)}(y)$ taken along a vanishing cycle $\gamma(y)$ whose ambiguity in homology class $H_{N}\left(X_{y}, \mathbf{Z}\right)$ we do not care for the moment,

$$
\begin{array}{r}
I_{\phi_{q}, \gamma(y)}(y):=\int_{\gamma(y)} \psi_{q}=\left(\frac{1}{2 \pi i}\right)^{K} \int_{\partial \gamma(y)} \frac{d f_{0} \wedge \ldots \wedge d f_{K-1} \wedge \psi_{q}}{\left(f_{0}-y_{0}\right) \cdots\left(f_{K-1}-y_{K-1}\right)} \\
=\left(\frac{1}{2 \pi i}\right)^{K} \int_{\partial \gamma(y)} \frac{\phi_{q}(u) d u}{\left(f_{0}-y_{0}\right) \cdots\left(f_{K-1}-y_{K-1}\right)},
\end{array}
$$

where $\partial \gamma(y)$ is a cycle of $H_{N}\left(X \backslash X_{y}\right)$ obtained by the aid of Leray's coboundary operator $\partial$. One may consult a booklet by F. Pham [15] or a book by V. A. Vassiliev [21] on the coboundary operator.

From (2.8) we can deduce

$$
\int_{\gamma(y)} \omega_{j}=\sum_{q=1}^{\mu\left(X_{0}\right)}\left[\sum_{i=0}^{K-1}(-1)^{i} p_{i} y_{i} P_{j q}^{(i)}(y)\right] I_{\phi_{q}, \gamma(y)}(y) .
$$

It is easily seen from the following evident equalities in view of definition (2.10),

$$
\begin{gathered}
\int_{\partial \gamma(y)} \frac{d f_{0} \wedge \ldots \wedge d f_{K-1}}{\left(f_{0}-y_{0}\right) \cdots\left(f_{K-1}-y_{K-1}\right)} \wedge d f_{i} \wedge i_{E}\left(\psi_{q}\right)=0, \quad 0 \leq i \leq K-1, \\
\int_{\partial \gamma(y)} \frac{d f_{0} \wedge \ldots \wedge d f_{K-1}}{\left(f_{0}-y_{0}\right) \cdots\left(f_{K-1}-y_{K-1}\right)} \wedge d \varphi=0, \quad \varphi \in \Omega_{X}^{N-1} .
\end{gathered}
$$

Let us compare (2.11) with the relation

$$
d \int_{\gamma(y)} \omega_{j}=\ell_{j} \sum_{q=1}^{\mu\left(X_{0}\right)}\left[\sum_{i=0}^{K-1}(-1)^{i} P_{j q}^{(i)}(y) d y_{i}\right] I_{\phi_{q}, \gamma(y)}(y),
$$

obtained from (2.7) and (2.11). As a result we get equations connecting $I_{\phi_{q}}(y)$ and $\frac{\partial}{\partial y_{\ell}} I_{\phi_{q}}$, $0 \leq \ell \leq K-1$ (we omit specifying $\gamma(y)$ except necessary cases),

$$
\begin{array}{r}
\frac{\partial}{\partial y_{\ell}}\left[\sum_{q=1}^{\mu\left(X_{0}\right)} \sum_{i=0}^{K-1}(-1)^{i} p_{i} y_{i} P_{j q}^{(i)} I_{\phi_{q}}\right]=\ell_{j} \sum_{q=1}^{\mu\left(X_{0}\right)}\left[\sum_{i=0}^{K-1}(-1)^{i} P_{j q}^{(i)}(y) d y_{i}\right] I_{\phi_{q}}(y), \\
1 \leq j \leq \mu\left(X_{0}\right) .
\end{array}
$$

Thus we have obtained a system of differential equations to be understood as the GaussManin connexion of the singularity $X_{0}$. To state the theorem in a simple form, we introduce the following notation: $\mathbf{I}_{V}=\left(\int_{\gamma(y)} \omega_{1}, \ldots, \int_{\gamma(y)} \omega_{\mu\left(X_{0}\right)}\right), \mathbf{I}_{\Phi}=\left(I_{\phi_{1}, \gamma(y)}(y), \ldots\right.$, $\left.\ldots, I_{\phi_{\mu\left(X_{0}\right)}, \gamma(y)}(y)\right)$, i.e. vectors of integrals taken along a certain vanishing cycle $\gamma(y)$. We define several other $\left(\mu\left(X_{0}\right) \times \mu\left(X_{0}\right)\right)$-matrices as follows:

$$
L_{V}=\operatorname{diag}\left(\ell_{1}, \ldots, \ell_{\mu\left(X_{0}\right)}\right)
$$

where $\ell_{i}=w\left(\omega_{i}\right)$, and $P^{(i)}(y)=\left(P_{j q}^{(i)}(y)\right)_{1 \leq j, q \leq \mu\left(X_{0}\right)}, i=0,1, \ldots, K-1$, are matrices consisting of elements defined in (2.6).

By summing up the above arguments and the theory due to Greuel [6], we obtain 
THEOREM 7.

1) For a quasihomogeneous mapping

$$
f: X \rightarrow Y
$$

with isolated complete intersection singularities of dimension $N$ like (2.1), the GaussManin system satisfied by $\mathbf{I}_{\Phi}$ is described as follows:

$$
d\left[\sum_{i=0}^{K-1}(-1)^{i} p_{i} y_{i} P^{(i)}(y) \mathbf{I}_{\Phi}\right]=L_{V}\left[\sum_{i=0}^{K-1}(-1)^{i} P^{(i)}(y) d y_{i}\right] \mathbf{I}_{\Phi} .
$$

2) The critical value $D$ (singular locus of the system (2.13)) of deformation $X_{y}$ is given by $D=\{y \in Y: \Delta(y)=0\}$ where

$$
\Delta(y)=\operatorname{det}\left(\sum_{i=0}^{K-1}(-1)^{i} p_{i} y_{i} P^{(i)}(y)\right) .
$$

Let us return to the problem (C.P.) of Section 1. Our main concern is to understand the integral (1.8) as a sum of integrals like (2.10) for a certain mapping $f$. To adapt our (C.P.) to the scheme explained before Theorem 7, we treat the mapping $f: X \rightarrow Y$ for $X=\left(\mathbf{C}_{u}^{n+\mu}, 0\right), Y=\left(\mathbf{C}_{y}^{\mu+1}, 0\right)$, defined by

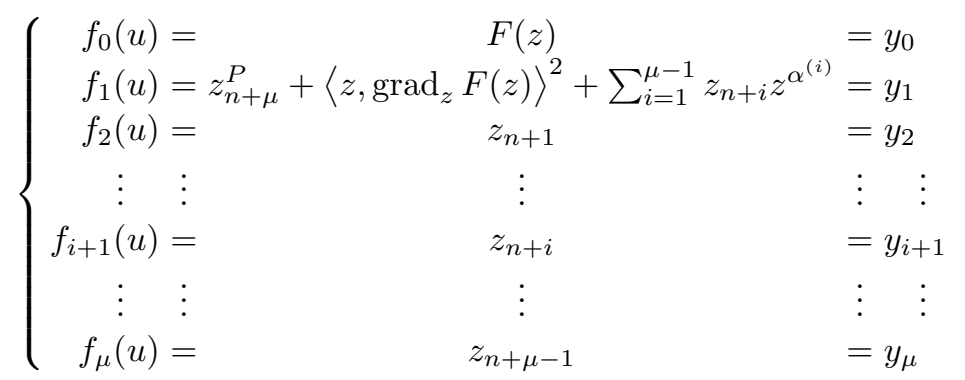

with the notation $z=\left(z_{1}, \ldots, z_{n}\right), z^{\prime}=\left(z_{n+1}, \ldots, z_{n+\mu-1}\right), u=\left(z, z^{\prime}, z_{n+\mu}\right)$. Here the power $P$ is an integer that corresponds to the denominator of $q \in \mathbf{Q}$.

LEMma 8. For $F(z)$ under conditions (C.2), (C.3), the mapping $\left(f_{0}, \ldots, f_{\mu}\right)$ given by (2.15) defines an isolated quasihomogeneous complete intersection singularity

$$
X_{0}=\left\{u \in X: f_{0}(u)=\ldots=f_{\mu}(u)=0\right\} .
$$

Namely,

$$
V=\frac{\Omega_{X}^{n+\mu}}{f_{0} \Omega_{X}^{n+\mu}+f_{1} \Omega_{X}^{n+\mu}+\sum_{i=1}^{\mu} z_{i+n} \Omega_{X}^{n+\mu}+d F \wedge \Omega_{X}^{n+\mu-1}+d f_{1} \wedge \Omega_{X}^{n+\mu-1}+\sum_{i=1}^{\mu} d z_{i+n} \wedge \Omega_{X}^{n+\mu-1}}
$$

is a finite-dimensional vector space.

Proof. The complete intersection property follows from the fact that two polynomials $F(z)$ and $\left\langle z, \operatorname{grad}_{z} F(z)\right\rangle$ are of the same quasihomogeneous weight but with different coefficients. This is a consequence of (C.2) 1) which supposes that $F(z)$ is not a homogeneous polynomial. The condition (C.3) entails immediately the finite dimensionality of $V$. 
To see that the components of $\mathbf{I}_{\Phi}$ defined for the mapping (2.15) give rise to integrals of type (1.8), we prepare

Lemma 9.

1) Let us define

$$
\Phi\left(z, z^{\prime}\right)=\left.\frac{\Omega_{X}^{n+\mu+1}}{d f_{0} \wedge \ldots d f_{\mu} \wedge \Omega_{X}^{n}+\sum_{i=0}^{\mu} f_{i} \Omega_{X}^{n+\mu+1}}\right|_{z_{n+\mu}=0} .
$$

Then the following natural isomorphism holds

$$
\Phi \cong \Phi\left(z, z^{\prime}\right) \otimes\left(\mathbf{C}\left[z_{n+\mu}\right] /\left\langle z_{n+\mu}^{P}\right\rangle\right) .
$$

2) For a Leray coboundary $\partial \gamma_{n-2} \in H_{N+K-1}\left(\left.X \backslash X_{y}\right|_{z_{n+\mu}=0}\right.$, Z) of a vanishing cycle $\gamma_{n-2} \in H_{n-2}\left(\left.X_{y}\right|_{z_{n+\mu}=0}, \mathbf{Z}\right)$ one can choose a corresponding vanishing cycle $\tilde{\gamma}_{n-1} \in$ $H_{n-1}\left(X_{y}, \mathbf{Z}\right)$ such that the equality

$$
\begin{aligned}
& \int_{\partial \gamma_{n-2}} \phi(z)\left(f _ { 1 } \left(z, y_{2}, \ldots,\right.\right.\left.\left.y_{\mu}, 0\right)-y_{1}\right)^{\frac{r+1}{P}-1} \frac{d z}{d F} \\
&=\epsilon\left(\frac{1}{2 \pi i}\right)^{\mu} \int_{\partial \tilde{\gamma}_{n-1}} \phi(z) z_{n+\mu}^{r} \frac{d u}{\left(f_{0}-u_{0}\right) \cdots\left(f_{\mu}-u_{\mu}\right)}
\end{aligned}
$$

holds, where $\epsilon \in \mathbf{C}^{\times}$such that $\epsilon^{P}=1$. Furthermore, the cycle $\partial \tilde{\gamma}_{n-1} \in H_{N+K}\left(X \backslash X_{y}\right)$ is homotopically equivalent to a product of a small circle on complex $z_{n+\mu}$-plane and $\partial \gamma_{n-2}$.

Proof. Statement 1) is evident. Statement 2) is an integral version of statement 1), which can be shown by means of equality (2.10).

Thus the singular locus of the integral (1.8) can be given by that of

$$
\int_{\tilde{\gamma}_{n-1}} \phi(z) z_{n+\mu}^{r} \frac{d u}{d f_{0} \wedge \ldots \wedge d f_{\mu}}
$$

with $\tilde{\gamma}_{n-1} \in H_{n-1}\left(X_{y}, \mathbf{Z}\right)$ after substitution $y_{1}=-W_{1}(x, t)$ (Case 1 after Lemma 3 ) or $y_{1}=0$ (Case 2 after Lemma 3$), y_{i}=W_{i}(x, t), 2 \leq i \leq \mu$. Let us remind that we denoted the quasihomogeneous weight of function $f_{i}$ by $p_{i}, 0 \leq i \leq \mu$. We define matrices $P^{(i)}(y)$, $2 \leq i \leq \mu$ for the mapping (2.15) after the master (2.6) and Theorem 7. Combining Theorem 7 with Lemma 9, we obtain

Theorem 10. The defining equation of the wave front (Proposition $1 \mathbf{c})$ ) is given by the polynomial

$$
\phi(x, t, s)=\left.\operatorname{det}\left(\sum_{i=0}^{\mu}(-1)^{i} p_{i} y_{i} P^{(i)}(y)\right)\right|_{y_{0}=s, y_{i}=W_{i}(x, t), 2 \leq i \leq \mu} .
$$

Here the restriction will be imposed in accordance with two cases considered just after Lemma 3, i.e. $y_{1}=-W_{1}(x, t)$ in Case 1 and $y_{1}=0$ in Case 2.

3. Fronts in the wave propagation on the plane. In this section we illustrate how effectively our machinery explained in Section 2 works in studying the wave propagation process on the plane (i.e. $n=2$ ). Our main argument consists in the fact that the integrals (1.8) can be expressed as sums of solutions to the Gauss-Manin system of $A_{\mu}$ 
type. We shall use further notation from the former sections if they can be understood without special modifications.

Let us suppose

$$
F(z)=z_{1}-G\left(z_{2}\right)
$$

for a polynomial of degree $k$ with real coefficients,

$$
G\left(z_{2}\right)=z_{2}^{k}+g_{1} z_{2}^{k-1}+g_{2} z_{2}^{k-2}+\ldots+g_{k-1} z_{2} .
$$

Without loss of generality, we can choose the initial wave front $S=\left\{x \in \mathbf{R}^{2}: F(x)=0\right\}$, i.e. $s=0$. As the phase function described in Proposition $1 \mathbf{c}$ ) restricted on $S$ we get

$$
\begin{aligned}
& \left.\psi(x, t, z)\right|_{S}=\left\langle\left(x_{1}-G\left(z_{2}\right), x_{2}-z_{2}\right),\left(-1, G^{\prime}\left(z_{2}\right)\right)\right\rangle^{2}-t^{2}\left(1+G^{\prime}\left(z_{2}\right)^{2}\right) \\
& \quad=(k-1)^{2}\left[z_{2}^{2 k}+y_{1}(x, t) z_{2}^{2 k-1}+y_{2}(x, t) z_{2}^{2 k-2}+\ldots+y_{2 k-1}(x, t) z_{2}+y_{2 k}(x, t)\right],
\end{aligned}
$$

where $y_{i}(x, t), 1 \leq i \leq 2 k$, are polynomials at most quadratic in $(x, t)$ variables defined as those which make (3.3) valid. More precisely, $y_{1}(x, t), \ldots, y_{k}(x, t)$ are linear in $x_{1}, x_{2}, t^{2}$. The remaining coefficients $y_{k+1}(x, t), \ldots, y_{2 k}(x, t)$ are linear in $x_{1}, x_{2}, t^{2}, x_{1} x_{2}, x_{1}^{2}, x_{2}^{2}$. Especially,

$$
\begin{gathered}
y_{1}(x, t)=(k-1)^{-2}\left(2 x_{2}+k-(2 k-1) g_{1}\right), \\
y_{2 k}(x, t)=(k-1)^{-2}\left(\left(x_{1}+g_{k-1} x_{2}\right)^{2}-t^{2}\left(1+g_{k-1}^{2}\right)\right) .
\end{gathered}
$$

Thus, in our situation the integral (1.8) is reduced to

$$
I_{\lambda}(y(x, t))=\int_{\gamma} H\left(G\left(z_{2}\right), z_{2}\right)\left[z_{2}^{2 k}+y_{1}(x, t) z_{2}^{2 k-1}+\ldots+y_{2 k}(x, t)\right]^{\lambda} d z_{2},
$$

for a certain vanishing cycle $\gamma$ of $\left.\psi(x, t, z)\right|_{S}=0$. Here we took into account the equivalence relation

$$
\frac{d z_{1} \wedge d z_{2}}{d F} \equiv d z_{2}
$$

Let us consider a series of integrals

$$
K_{i}^{\lambda}(y)=\int_{\gamma} z^{i} f_{1}(y, z)^{\lambda} d z, \quad i=0, \ldots, 2 k+1,
$$

where we used the notation

$$
f_{1}(y, z):=\left.(k-1)^{-2} \psi(x, t, z)\right|_{S}=z_{2}^{2 k}+y_{1} z_{2}^{2 k-1}+y_{2} z_{2}^{2 k-2}+\ldots+y_{2 k} .
$$

It is evident that our object of investigation (3.4) can be interpreted as a sum of the integrals (3.5) with $\mathbf{C}[y]$ coefficients. That is to say, the singular locus $D=\{y \in Y: \Delta(y)=0\}$ where $\Delta(y)$ is the discriminant of $f_{1}(y, z)$ coincides with the singular support of (3.4) if we consider the latter as a function in $y$ variables. Thus the analysis of integrals (3.5) entails not only geometric but also analytic properties of $C^{\infty}$ singularities of (3.4).

We remind the following statement that gives complete description of the overdetermined system (the Gauss-Manin connexion with $A_{2 k-1}$ singularities) satisfied by the integrals $K_{i}^{\lambda}(y)$. 
THEOREM 11 ([12], [13]).

1) The integrals $K_{0}^{\lambda}(y), \ldots, K_{4 k-2}^{\lambda}(y)$ satisfy the following over-determined system of differential equations:

$$
\begin{gathered}
\sum_{\ell=0}^{2 k-1} y_{2 k-\ell} \frac{\partial}{\partial y_{2 k-\ell}} K_{i}^{\lambda}+\frac{\partial}{\partial y_{2 k}} K_{2 k+i}^{\lambda}=\lambda K_{i}^{\lambda}, \quad 0 \leq i \leq 2 k-2, \\
\sum_{\ell=1}^{2 k-1} \ell y_{2 k-\ell} \frac{\partial}{\partial y_{2 k-\ell}} K_{j}^{\lambda}+2 k \frac{\partial}{\partial y_{2 k}} K_{2 k+j}^{\lambda}=-(j+1) K_{j}^{\lambda}, \quad-1 \leq j \leq 2 k-2 .
\end{gathered}
$$

2) In the form of matrix, the above systems (3.6), (3.7) admit an expression

$$
\Pi(y) \cdot \vec{b}=\vec{a}
$$

where

$$
\begin{aligned}
& \Pi(y)=\left[\begin{array}{cccccccccc}
y_{2 k} & y_{2 k-1} & \cdots & y_{2} & y_{1} & 1 & \cdots & 0 & 0 & 0 \\
0 & y_{2 k} & \cdots & y_{3} & y_{2} & y_{1} & \cdots & 0 & 0 & 0 \\
0 & 0 & \cdots & y_{4} & y_{3} & y_{2} & \cdots & 0 & 0 & 0 \\
\vdots & \vdots & \ddots & \vdots & \vdots & \vdots & \cdots & \vdots & \vdots & \vdots \\
0 & 0 & \cdots & y_{2 k-1} & y_{2 k-2} & y_{2 k-3} & \cdots & y_{1} & 1 & 0 \\
0 & 0 & \cdots & y_{2 k} & y_{2 k-1} & y_{2 k-2} & \cdots & y_{2} & y_{1} & 1 \\
y_{2 k-1} & 2 y_{2 k-2} & \cdots & (2 k-1) y_{1} & 2 k & 0 & \cdots & 0 & 0 & 0 \\
0 & y_{2 k-1} & \cdots & (2 k-2) y_{2} & (2 k-1) y_{1} & 2 k & \cdots & 0 & 0 & 0 \\
\vdots & \vdots & \cdots & \vdots & \vdots & \vdots & \ddots & \vdots & \vdots & \vdots \\
0 & 0 & \cdots & 2 y_{2 k-2} & 3 y_{2 k-3} & 4 y_{2 k-4} \cdots & 2 k & 0 & 0 \\
0 & 0 & \cdots & y_{2 k-1} & 2 y_{2 k-2} & 3 y_{2 k-3} & \cdots & (2 k-1) y_{1} & 2 k & 0 \\
0 & 0 & \cdots & 0 & y_{2 k-1} & 2 y_{2 k-2} & \cdots & (2 k-2) y_{2}(2 k-1) y_{1} & 2 k
\end{array}\right] \\
& \vec{a}=\left(\lambda K_{0}^{\lambda}, \lambda K_{1}^{\lambda}, \ldots, \lambda K_{2 k-2}^{\lambda}, 0,-K_{0}^{\lambda},-2 K_{1}^{\lambda}, \ldots,-(2 k-1) K_{2 k-2}^{\lambda}\right)^{T}, \\
& \vec{b}=\left(\frac{\partial}{\partial y_{2 k}} K_{0}^{\lambda}, \frac{\partial}{\partial y_{2 k}} K_{1}^{\lambda}, \ldots, \frac{\partial}{\partial y_{2 k}} K_{4 k-2}^{\lambda}\right)^{T} .
\end{aligned}
$$

To be remarked, $\frac{\partial}{\partial y_{2 k-i}} K_{j}^{\lambda}(y)=\frac{\partial}{\partial y_{2 k-j}} K_{i}^{\lambda}(y)$.

In [18] we derived a partial differential operator of Fuchsian type annihilating $K_{j}^{\lambda}(y)$.

By the aid of the above theorem, we get our main result on 2-dimensional wave propagation.

Let us denote by $\Delta(y)$ the discriminant of $f_{1}(z, y)$ calculated by

$$
\Delta(y)=\operatorname{det} \Pi(y),
$$

for $\Pi(y)$ defined in (3.8). In the literature $\Pi(y)$ is often called the resultant of Sylvester.

Theorem 12.

1) The wave front $\Sigma$ of (C.P.) with the initial front $S=\left\{x \in \mathbf{R}^{2}: F(x)=0\right\}$ given by (3.1), (3.2), admits the expression

$$
\Sigma=\left\{(x, t) \in \mathbf{R}^{3}: \Delta(y(x, t))=0\right\}
$$

where $y(x, t)=\left(y_{1}(x, t), \ldots, y_{2 k}(x, t)\right)$ is defined in $(3.3)$. 
2) Except ordinary double points, $\Sigma$ has cusps of type at most $(2 k-1,8 k-2)$. In other words, if under a suitable local diffeomorphism $\left(x_{1}, x_{2}\right) \mapsto\left(X_{1}, X_{2}\right)$ the restriction of wave front at a certain moment $\left.\Sigma\right|_{t=\text { const. }}$ admits as its local equation

$$
\left\{X_{1}(x)^{p_{1}}-X_{2}(x)^{p_{2}}=0\right\}, \quad p_{1} \leq p_{2},
$$

then these powers satisfy $p_{1} \leq 2 k-1, p_{2} \leq 8 k-2$.

Proof. Statement 1) follows if one applies Theorem 11 to our integral (3.5), and then replaces variables $y$ by $y(x, t)$ defined in $(3.3)$.

To see (3.10), one will examine the matrix $\Pi(y)$. After $(3.8), \Delta(y)=\operatorname{det} \Pi(y)$ contains the term $y_{2 k}^{2 k-1}$ highest in variable $y_{2 k}$, while $\Delta(y)$ is quasihomogeneous with respect to the weight $w\left(y_{i}\right)=i, 1 \leq i \leq 2 k$. According to this weight, every term of $\Delta(y)$ must be of weight $2 k(2 k-1)$. Remark that

$$
y_{2 k}(x, t)=L(x, t) L(x,-t)
$$

for

$$
L(x, t)=(k-1)^{-1}\left(x_{1}+g_{k-1} x_{2}-\left(1+g_{k-1}^{2}\right)^{1 / 2} t\right) .
$$

Hence $L(x, t) \neq L(x,-t)$ out of $t=0$ and $y_{2 k}(x, t)$ can never be a constantly zero function for any choice of $\left(g_{1}, \ldots, g_{k-1}\right)$.

The terms that may appear in the expression $\Delta(y)$ must be of degree less than $4 k-1$ after concrete form of $\Pi(y)$. According to the remark after $(3.3), y_{i}(x, t)$ are at most quadratic polynomials. Suppose that there exists a set of indices $1 \leq \alpha_{j} \leq 2 k-1$, $\left\{\alpha_{1}, \ldots, \alpha_{\ell}\right\} \subset\{1, \ldots, 2 k-1\}$ such that

$$
y_{\alpha_{j}}(x, t)=c_{\alpha_{j}} X^{2}(x, t)
$$

for a linear function $X(x, t)$. Such cases do really happen if the coefficients of $G\left(z_{2}\right)$, $\left(g_{1}, \ldots, g_{k-1}\right)$ satisfy certain algebraic equations of degree 4 (see Remark 1 below). Suppose that for $X(x, t)$ in question such a set $\left\{\alpha_{1}, \ldots, \alpha_{M}\right\}$ is maximal, i.e. for complementary indices $\left\{\beta_{1}, \ldots, \beta_{2 k-M-1}\right\}=\{1, \ldots, 2 k-1\} \backslash\left\{\alpha_{1}, \ldots, \alpha_{M}\right\}, y_{\beta_{i}}(x, t), 1 \leq i \leq$ $2 k-M-1$ are not divided by $X^{2}(x, t)$. Then evidently,

$$
\Delta(y)=y_{2 k}^{2 k-1}-\text { const. } X^{2(4 k-1)}(x, t)+R\left(X, y_{\beta_{1}}, \ldots, y_{\beta_{2 k-M-1}}, y_{2 k}\right),
$$

where $R\left(X, y_{\beta_{1}}, \ldots, y_{\beta_{2 k-M-1}}, y_{2 k}\right)$ is a polynomial of degree not exceeding $2(4 k-2)$ in $X$ and not greater than $(4 k-2)$ in $y_{\beta_{1}}, \ldots, y_{\beta_{2 k-M-1}}, y_{2 k}$. The coefficients of the polynomial $R$ can vary in dependence of $\left(g_{1}, \ldots, g_{k-1}\right)$. From the above expression of $\Delta(y)$ statement 2 ) follows.

R e mark 1 . On the divisibility of a certain $y_{\alpha_{j}}(x, t)$ by a square of another function, one can calculate, in principle, the condition to be satisfied by $\left(g_{1}, \ldots, g_{k-1}\right)$. For example a sufficient condition so that $y_{\ell}\left(x, t_{0}\right), \ell<k$ be expressed as a square of a linear function in $x_{2}$ at $t=t_{0} \neq 0$, sounds

$$
\begin{aligned}
& \left(\sum_{i+j=\ell-1}(2 k-\ell-2(k-i)(k-j)) g_{i} g_{j}\right)^{2}=4\left[\sum_{i+j=\ell-2}(k-i)(k-j) g_{i} g_{j}\right] \\
& \quad \times\left[\sum_{i+j=\ell}(2(k-i)(k-j)-2 k+\ell+1) g_{i} g_{j}-t_{0}^{2} \sum_{i+j=\ell-2}(k-i)(k-j) g_{i} g_{j}\right] .
\end{aligned}
$$


Conditions corresponding to other cases have a similar form. We omit them in view of purely technical character of such conditions.

4. An illustration by Gauss' hypergeometric function. In this section, we mention a little about analytic aspects of our theory. Namely we illustrate how to determine sharp(-diffuse) type at singular locus of fronts $((2,3)$ cusp) in an adequate way by means of the Gauss-Manin system of $A_{2}$ type that admits solutions expressed through Gauss' hypergeometric functions.

We say that a distribution $u(x)$ with $C^{\infty}$ singular locus on $\Sigma=\{x: \phi(x)=0\}$ has the sharp (resp. diffuse) front from the side $\Sigma_{+}=\{x: \phi(x)>0\}$ in approaching to a point of $\Sigma$ if and only if there exists (resp. does not exist) a $C^{\infty}$ extension of $u(x)$ across $\Sigma$. The distribution $u(x)$ has lacuna inside $\Sigma_{+}$iff $u(x) \equiv 0$ on $\Sigma_{+}$, see [14]. This notion has been introduced in connexion with the situation that a diffuse front can never be a front of a lacuna component. On the other hand, sharpness of a front is a necessary condition for presence of lacuna. For details and typical examples see [5].

Though the degree of a phase function $f_{1}(y(x, t), z)$ defined in (3.3) cannot be odd, an observation in terms of $A_{2}$ type singularity is useful because of the adjacency property of $A_{2}$ singularity to almost all more complicated singularities. In other words, one can expect that the solutions to (C.P.) are expressed by means of period integrals of $A_{2}$ type around the generic points on the singular loci of the wave front. Let us look at the integrals

$$
K_{i}^{\lambda}(y)=\int_{\Gamma} z^{i}\left(z^{3}+y_{1} z+y_{0}\right)^{\lambda} d z, \quad i=0,1,
$$

where $\Gamma$ is a double loop of Pochhammer (cf. [2]), i.e. a one-dimensional regularized cycle. Then from Theorem 11 (or from [18]) one can deduce the following differential equations for $K_{0}^{\lambda}(y), K_{1}^{\lambda}(y)$.

$$
\begin{aligned}
& {\left[\left(27 y_{0}^{2}+4 y_{1}^{3}\right)\left(\frac{\partial}{\partial y_{0}}\right)^{2}+27(1-2 \lambda) y_{0} \frac{\partial}{\partial y_{0}}+27\left(\lambda^{2}-1 / 9\right)\right] K_{0}^{\lambda}(y)=0} \\
& {\left[\left(27 y_{0}^{2}+4 y_{1}^{3}\right)\left(\frac{\partial}{\partial y_{0}}\right)^{2}+27(1-2 \lambda) y_{0} \frac{\partial}{\partial y_{0}}+27\left(\lambda^{2}-4 / 9\right)\right] K_{1}^{\lambda}(y)=0 .}
\end{aligned}
$$

The equation $(4.1)_{0}$ has two independent solutions $U_{1, \lambda}(y), U_{2, \lambda}(y)$ expressed by Gauss' hypergeometric function:

$$
\begin{aligned}
& U_{1, \lambda}(y)=y_{1} y_{0}^{-\lambda+1 / 3} \cdot{ }_{2} F_{1}\left(\frac{-\lambda}{2}-\frac{1}{6}, \frac{-\lambda}{2}+\frac{1}{3}, \frac{2}{3} ; \frac{-4 y_{1}^{3}}{27 y_{0}^{2}}\right) \\
& U_{2, \lambda}(y)=y_{1}^{2} y_{0}^{-\lambda-1 / 3} \cdot{ }_{2} F_{1}\left(\frac{-\lambda}{2}+\frac{1}{6}, \frac{-\lambda}{2}+\frac{2}{3}, \frac{4}{3} ; \frac{-4 y_{1}^{3}}{27 y_{0}^{2}}\right) .
\end{aligned}
$$

The solutions to $(4.1)_{1}$ are written down in a similar way. As these two solutions $U_{1, \lambda}(y), U_{2, \lambda}(y)$ correspond to two independent basis $\gamma_{1}, \gamma_{2}$ of the twisted homology group, we can write

$$
U_{j, \lambda}^{\epsilon}(y)=\int_{\gamma_{j}} \chi_{\lambda}^{\epsilon}\left(z^{3}+y_{1} z+y_{0}\right) d z \quad(j=1,2) .
$$


These solutions ramify around the singular locus $\Sigma=\left\{y \in \mathbf{R}^{2}: \Delta_{2}(y)=0\right\}$. Here $\Delta_{2}(y)=27 y_{0}^{2}+4 y_{1}^{3}$. We shall remark that $\int_{\gamma_{i}} z^{j} \chi_{\lambda}^{\epsilon}\left(z^{3}+y_{1} z+y_{0}\right) d z$ and $U_{i, \lambda}^{\epsilon}(y)$ have the same sharp-diffuse type because of the relation

$$
\left(\frac{\partial}{\partial y_{1}}\right)^{j} U_{i, \lambda}^{\epsilon}(y)=\left(\frac{\partial}{\partial y_{0}}\right)^{j} \int_{\gamma_{i}} z^{j} \chi_{\lambda}^{\epsilon}\left(z^{3}+y_{1} z+y_{0}\right) d z
$$

It will be noted that the sharp-diffuse type does not change by differentiation or integration of a distribution. To study the asymptotic behaviour of $U_{i, \lambda}^{\epsilon}$ around $\Sigma$, we remind here the connexion formula for Gauss' hypergeometric function:

$$
\begin{aligned}
& { }_{2} F_{1}(\alpha, \beta, \gamma ; x)=\frac{\Gamma(\gamma) \Gamma(\gamma-\alpha-\beta)}{\Gamma(\gamma-\alpha) \Gamma(\gamma-\beta)} \cdot{ }_{2} F_{1}(\alpha, \beta, \alpha+\beta-\gamma+1 ; 1-x) \\
& \quad+\frac{\Gamma(\gamma) \Gamma(\alpha+\beta-\gamma)}{\Gamma(\alpha) \Gamma(\beta)}(1-x)^{\gamma-\alpha-\beta} \cdot{ }_{2} F_{1}(\gamma-\alpha, \gamma-\beta, \gamma-\alpha-\beta+1 ; 1-x) .
\end{aligned}
$$

These are based on a relation between 4 expressions from Kummer's 24 integrals, that goes back to Gauss (cf. [2, Chapitre I (10)]). By applying this connexion formula to our $U_{1, \lambda}(y)$ and $U_{2, \lambda}(y)$, we get

$$
\begin{aligned}
{ }_{2} F_{1} & \left(-\frac{\lambda}{2}-\frac{1}{6},-\frac{\lambda}{2}+\frac{1}{3}, \frac{2}{3} ; \frac{-4 y_{1}^{3}}{27 y_{0}^{2}}\right) \\
= & \frac{\Gamma\left(\frac{2}{3}\right) \Gamma\left(\lambda+\frac{1}{2}\right)}{\Gamma\left(\frac{\lambda}{2}+\frac{5}{6}\right) \Gamma\left(\frac{\lambda}{2}+\frac{1}{3}\right)} \cdot{ }_{2} F_{1}\left(-\frac{\lambda}{2}-\frac{1}{6},-\frac{\lambda}{2}+\frac{1}{3},-\lambda+\frac{1}{2} ; 1+\frac{4 y_{1}^{3}}{27 y_{0}^{2}}\right) \\
& +\frac{\Gamma\left(\frac{2}{3}\right) \Gamma\left(-\lambda-\frac{1}{2}\right)}{\Gamma\left(-\frac{\lambda}{2}-\frac{1}{6}\right) \Gamma\left(-\frac{\lambda}{2}+\frac{1}{3}\right)}\left(1+\frac{4 y_{1}^{3}}{27 y_{0}^{2}}\right)^{\lambda+\frac{1}{2}} \cdot{ }_{2} F_{1}\left(\frac{\lambda}{2}+\frac{5}{6}, \frac{\lambda}{2}+\frac{1}{3}, \lambda+\frac{3}{2} ; 1+\frac{4 y_{1}^{3}}{27 y_{0}^{2}}\right), \\
{ }_{2} F_{1}\left(-\frac{\lambda}{2}+\frac{1}{6},-\frac{\lambda}{2}+\frac{2}{3}, \frac{4}{3} ; \frac{-4 y_{1}^{3}}{27 y_{0}^{2}}\right) & \frac{\Gamma\left(\frac{4}{3}\right) \Gamma\left(\lambda+\frac{1}{2}\right)}{\Gamma\left(\frac{\lambda}{2}+\frac{3}{2}\right) \Gamma\left(\frac{\lambda}{2}+\frac{2}{3}\right)} \cdot{ }_{2} F_{1}\left(-\frac{\lambda}{2}+\frac{1}{6},-\frac{\lambda}{2}+\frac{2}{3},-\lambda+\frac{1}{2} ; 1+\frac{4 y_{1}^{3}}{27 y_{0}^{2}}\right) \\
& +\frac{\Gamma\left(\frac{4}{3}\right) \Gamma(-\lambda-12)}{\Gamma\left(-\frac{\lambda}{2}+\frac{1}{6}\right) \Gamma\left(-\frac{\lambda}{2}+\frac{2}{3}\right)}\left(1+\frac{4 y_{1}^{3}}{27 y_{0}^{2}}\right)^{\lambda+\frac{1}{2}} \cdot{ }_{2} F_{1}\left(\frac{\lambda}{2}+\frac{3}{2}, \frac{\lambda}{2}+\frac{2}{3}, \lambda+\frac{3}{2} ; 1+\frac{4 y_{1}^{3}}{27 y_{0}^{2}}\right) .
\end{aligned}
$$

It is evident that the second term in the above expressions ramifies around the singular locus $\Sigma$, if $\lambda$ is not a half integer. Hence we obtain the following four classifications of sharp-diffuse type. We write down here the classification only for $U_{1, \lambda}^{\epsilon}$ while $U_{2, \lambda}^{\epsilon}$ behaves in a parallel manner.

Case 1. $\lambda=-1, \epsilon=-1$.

$$
\begin{array}{ll}
U_{1,-1}^{-}(y)=0 & \text { if } y_{0}>0, \Delta_{2}(y)>0, \\
U_{1,-1}^{-}(y)=\text { const. } y_{1}\left|y_{0}\right|^{4 / 3} \cdot{ }_{2} F_{1}\left(\frac{1}{3}, \frac{5}{6}, \frac{2}{3} ;-\frac{4 y_{1}^{3}}{27 y_{0}^{2}}\right) & \text { if } y_{0}<0, \Delta_{2}(y)>0, \\
U_{1,-1}^{-}(y)=\text { const. } y_{1}\left|y_{0}\right|^{7 / 3} \Delta_{2}(y)^{-1 / 2} \cdot{ }_{2} F_{1}\left(1, \frac{1}{6}, \frac{1}{2} ; 1+\frac{4 y_{1}^{3}}{27 y_{0}^{2}}\right) & \text { if } y_{0} \neq 0, \Delta_{2}(y)<0 .
\end{array}
$$


Hence $U_{1,-1}^{-}(y)$ has the sharp front in approaching to a generic point on $\Sigma$ from the side $\Delta_{2}(y)>0$, while it has the diffuse front in approaching to the singular point of $\Sigma$ (i.e. the origin). It has the diffuse front from the side $\Delta_{2}(y)<0$.

Case 2. $\lambda=-1, \epsilon=+1$.

$$
\begin{aligned}
& U_{1,+1}^{-}(y)=\text { const. } y_{1}\left|y_{0}\right|^{7 / 3} \Delta_{2}(y)^{-1 / 2} \cdot{ }_{2} F_{1}\left(1, \frac{1}{6}, 1 ; 1+\frac{4 y_{1}^{3}}{27 y_{0}^{2}}\right)+\operatorname{hol}\left(1+\frac{4 y_{1}^{3}}{27 y_{0}^{2}}\right) \\
& U_{1,+1}^{-}(y)=0 \\
& \text { if } y_{0} \neq 0, \Delta_{2}(y)>0 \text {, } \\
& \text { if } \Delta_{2}(y)<0 \text {. }
\end{aligned}
$$

(here $\operatorname{hol}(z)$ denotes a holomorphic function in the neighbourhood of $z=0$ ).

Hence $U_{1,+1}^{-}(y)$ has the diffuse front in approaching to $\Sigma$ from the side $\Delta_{2}(y)>0$. It has always lacuna (a fortiori sharp front) from the side $\Delta_{2}(y)<0$.

Case 3. $\lambda=-\frac{3}{2}, \epsilon=-1$.

$$
\begin{array}{ll}
U_{1,-3 / 2}^{-}(y)=0 & \text { if } y_{0}>0 \\
U_{1,-3 / 2}^{-}(y)=\text { const. } y_{1}\left|y_{0}\right|^{11 / 6} \Delta_{2}(y)^{-1} \cdot{ }_{2} F_{1}\left(\frac{3}{4}, \frac{-1}{12}, 0 ; 1+\frac{4 y_{1}^{3}}{27 y_{0}^{2}}\right) & \text { if } y_{0}<0 .
\end{array}
$$

Hence $U_{1,-3 / 2}^{-}(y)$ has the sharp front in approaching to a generic point of $\Sigma$ from any side if $y_{0}>0$. It has the diffuse front from any side of $\Sigma$ if $y_{0}<0$. In approaching to a singular point of $\Sigma, U_{1,-3 / 2}^{-}(y)$ has always the diffuse front.

Case 4. $\lambda=-\frac{3}{2}, \epsilon=+1$.

$$
\begin{aligned}
& U_{1,-3 / 2}^{+}(y)=c_{1} y_{1} y_{0}^{11 / 6} \text { p.v. }(1+\left.\frac{4 y_{1}^{3}}{27 y_{0}^{2}}\right)^{-1} \cdot{ }_{2} F_{1}\left(\frac{3}{4}, \frac{-1}{12}, 0 ; 1+\frac{4 y_{1}^{3}}{27 y_{0}^{2}}\right) \\
&+c_{2} y_{1} y_{0}^{11 / 6} \cdot{ }_{2} F_{1}\left(\frac{7}{12}, \frac{13}{12}, 2 ; 1+\frac{4 y_{1}^{3}}{27 y_{0}^{2}}\right) \text { if } y_{0}>0, \\
& U_{1,-3 / 2}^{+}(y)=c_{1}^{\prime} y_{1} y_{0}^{11 / 6} \delta\left(1+\frac{4 y_{1}^{3}}{27 y_{0}^{2}}\right) \cdot{ }_{2} F_{1}\left(\frac{3}{4}, \frac{-1}{12}, 0 ; 1+\frac{4 y_{1}^{3}}{27 y_{0}^{2}}\right) \\
&+c_{2}^{\prime} y_{1} y_{0}^{11 / 6} \cdot{ }_{2} F_{1}\left(\frac{7}{12}, \frac{13}{12}, 2 ; 1+\frac{4 y_{1}^{3}}{27 y_{0}^{2}}\right) \text { if } y_{0}<0
\end{aligned}
$$

(here p.v. denotes the principal value distribution of J. Hadamard). Hence we conclude that $U_{1,-3 / 2}^{+}(y)$ has the diffuse front in approaching to a generic point of $\Sigma$ from any side if $y_{0}>0$. It has the sharp front from any side of $\Sigma$ if $y_{0}<0$. In approaching to a singular point of $\Sigma, U_{1,-3 / 2}^{-}(y)$ has always the diffuse front.

Remark 2. The above results yield not only known pictures but also add supplementary details to the figures drawn in [5]. For example we can add an arrow " $d$ " (indicating the diffuse front) directed to the singular point of cusp from the right side to Figure 3. $\lambda=+1, \epsilon=-1$. Furthermore, we can add two arrows " $s$ " directed to the singular points appearing in a swallow tail ("back bone") from the inside to Figure 5. integer, + . 


\section{References}

[1] A. G. Aleksandrov and S. Tanabé, Gauss-Manin connexions, logarithmic forms and hypergeometric functions, in: Geometry from the Pacific Rim (Singapore 1994), Walter de Gruyter, Berlin, 1997, 1-21.

[2] P. Appell and J. Kampé de Fériet, Fonctions hypergeometriques et hypersphériques, Gauthier-Villars, Paris, 1926.

[3] M. F. Atiyah, R. Bott, L. Gårding, Lacunas for hyperbolic differential operators with constant coefficients, II, Acta Math. 131 (1973), 145-206.

[4] E. Brieskorn, Die Monodromie der isolierten Singularitäten von Hyperflächen, Manuscripta Math. 2 (1970), 103-161.

[5] L. Gårding, Sharp fronts of paired oscillatory integrals, Publ. Res. Inst. Math. Sci. 12 (1976/77), suppl., 53-68; Corrections: Publ. Res. Inst. Math. Sci. 13 (1977/78), 821.

[6] G.-M. Greuel, Der Gauß-Manin Zusammenhang isolierter Singularitäten von vollständigen Durchschnitten, Math. Ann. 214 (1975), 235-266.

[7] G.-M. Greuel, H. A. Hamm, Invarianten quasihomogener vollständiger Durchschnitte, Invent. Math. 49 (1978), 67-86.

[8] Y. Hamada, The singularities of the solutions of the Cauchy problem, Publ. Res. Inst. Math. Sci. 5 (1969), 21-40.

[9] Y. Hamada, J. Leray, C.Wagschal, Systèmes d'équations aux dérivées partielles à caractéristiques multiples: problème de Cauchy ramifié; hyperbolicité partielle, J. Math. Pures Appl. (9) 55 (1976), 297-352.

[10] L. Hörmander, The Analysis of Linear Partial Differential Operators, vol. I Grundlehren Math. Wiss. 256, Springer, Berlin, 1983.

[11] E. Leichtnam, Le problème de Cauchy ramifié linéaire pour des données à singularités algébriques, Mém. Soc. Math. France 53 (1993), 128 pp.

[12] Kh. M. Malikov, Over-determination of a system of differential equations for versal integrals of type A, D, E (in Russian), Differ. Uravn. 18 (1982), 1394-1401; English transl.: Differential Equations 18 (1982), 986-991.

[13] V. P. Palamodov, Deformations of complex spaces, in: Several Complex Variables IV, Encyclopaedia Math. Sci. 10, Springer, Berlin, 1990, 105-194.

[14] I. G. Petrovskiŭ, On the diffusion of waves and the lacunas for hyperbolic equations, Mat. Sb.(N.S.) 17(59) (1945), 289-370.

[15] F. Pham, Introduction à l'étude topologique des singularités de Landau, Gauthier-Villars, Paris, 1967.

[16] S. Tanabé, Lagrangian variety and the condition for the presence of sharp front of the fundamental solution to Cauchy problem, Sci. Papers College Arts Sci. Univ. Tokyo 42 (1992), 149-159.

[17] S. Tanabé, Transformée de Mellin des intégrales fibres de courbe espace associées aux singularités isolées d'intersection complète quasihomogènes, preprint MPIM Bonn, 1998, $28 \mathrm{pp}$.

[18] S. Tanabé, Connexion de Gauss-Manin associée à la déformation verselle des singularités isolées d'hypersurface et son application au XVIe problème de Hilbert, Ann. Institut Fourier (Grenoble), submitted.

[19] S. Tanabé, An application of Gauss-Manin systems to the asymptotic analysis around singular locus in wave propagations, Ann. Institut Fourier (Grenoble), submitted.

[20] A. N. Varchenko, On normal forms of nonsmoothness of solutions of hyperbolic equations, Izv. Akad. Nauk SSSR Ser. Mat. 51 (1987), 652-665; English transl.: Math. USSR-Izv. 30 (1988), 615-628.

[21] V. A. Vassiliev, Ramified Integrals, Singularities and Lacunas, Math. Appl. 315, Kluwer Acad. Publ., Dordrecht, 1995. 